\title{
Epidemiology and outcome of sepsis in adult patients with Streptococcus pneumoniae infection in a Norwegian county 1993-2011: an observational study
}

Åsa Askim ${ }^{1,2,10,11^{*}}$, Arne Mehl ${ }^{3,4,10}$, Julie Paulsen ${ }^{3,4,10}$, Andrew T. DeWan ${ }^{8}$, Didrik F. Vestrheim ${ }^{9}$, Bjørn Olav Åsvold ${ }^{5,7,10}$, Jan Kristian Damås ${ }^{3,6,10}$ and Erik Solligård ${ }^{1,2,10}$

\begin{abstract}
Background: Invasive pneumococcal disease (IPD) is responsible for significant mortality and morbidity worldwide. There are however few longitudinal studies on the changes in case fatality rate of IPD in recent years. We carried out a prospective observational study of patients with IPD in Nord Trøndelag county in Norway from 1993 to 2011 to study the clinical variables and disease outcome. The main outcome was all-cause mortality after 30 and 90 days.

Methods: Patients with positive blood cultures were registered prospectively by the microbiology laboratory and clinical variables were registered retrospectively from patients' hospital records. The severity of sepsis was assigned according to the 2001 International Sepsis Definition Conference criteria. The association between mortality and predictive factors was studied using a logistic regression model.

Results: The total number of patients was 414 with mean age of 67 years and $53 \%$ were male. Comorbidity was assessed by the Charlson Comorbidity Index (CCI). A CCl-score of 0 was registered in 144 patients (34.8\%), whereas 190 had a score of $1-2(45.9 \%)$ and $80(19.3 \%)$ had a score $\geq 3.68 .8 \%$ of the patients received appropriate antibiotics within the first $6 \mathrm{~h}$. The 30-day mortality risk increased by age and was 3 -fold higher for patients aged $\geq 80$ years ( $24.9,95 \% \mathrm{Cl} 16.4-33.4 \%)$ compared to patients aged $<70$ (8.0, $95 \% \mathrm{Cl} 3.5-12.4 \%) .110$ patients, (26.6\%) had severe sepsis and 37 (8.9\%) had septic shock. The 30 day all-cause mortality risk for those with sepsis without organ failure was $5.4 \%$ (95\% Cl 2.7-8.0 \%), $20.2 \%$ (95 \% Cl 13.5-27.4\%) for those with severe sepsis and $35.0 \%$ (95 \% Cl 21.6-49.0 \%) for those with septic shock. The mortality risk did not differ between the first and the second halves of the study period with a 30-day mortality risk of $13.5 \%$ (95 \% Cl 7.9-19.2\%) for 1993-2002 versus $11.8 \%$ (95 \% Cl 8.2-15.3\%) for 2003-2011.

Conclusion: IPD carries a high mortality despite early and appropriate antibiotics in most cases. We found no substantial decrease in case fatality rate during the study period of 18 years. Older age and higher severity of disease were important risk factors for death in IPD.
\end{abstract}

Keywords: Streptococcus pneumoniae, Bacteremia, Sepsis

\footnotetext{
* Correspondence: asa.askim@ntnu.no

${ }^{1}$ Clinic of Anaesthesia and Intensive Care, St Olav University Hospital,

Trondheim, Norway

${ }^{2}$ Department of Circulation and Medical Imaging, Norwegian University of

Science and Technology, Trondheim, Norway

Full list of author information is available at the end of the article
} 


\section{Background}

Streptococcus pneumonia colonizes the upper airway in approximately $10 \%$ of adults and is responsible for the majority of episodes of community-acquired pneumonia $[1,2]$. It may penetrate beyond epithelial cells and cause invasive disease and bacteremia [3]. Invasive pneumococcal disease (IPD) and sepsis are responsible for substantial morbidity and mortality worldwide with about 2 million deaths each year [4]. The case fatality rate of IPD and sepsis is $11-30 \%$ in studies in Europe and the US [5]. The incidence of IPD is highest in the $<2$ and $>65$ year age groups and in the older population, chronic comorbid disease is a risk factor for acquiring IPD [2]. The 23-valent pneumococcal polysaccharide vaccine (PPV23) has been recommended to risk-groups and adults aged $\geq 65$ years since 1996. The uptake is limited, and has been estimated to be about 15-30\% [6]. The 7-valent pneumococcal conjugate vaccine (PCV7) was introduced in the childhood immunization program in July 2006, and was replaced by the 13-valent vaccine (PCV13) in 2011. Since 2009, the uptake has been about $92 \%$ for the complete immunization schedule [7].

The development of fatal pneumococcal sepsis depends on a complex interplay between the host and the microbe $[8,9]$. Host factors such as diabetes, liver disease and COPD are associated with increased case fatality rate among immunocompetent patients. In the immunocompromised patients, HIV-infection, chronic renal failure and malignant disease have been associated with increased case fatality [10, 11]. Various guidelines have been developed to describe the optimal management of IPD and sepsis, such as the Surviving sepsis campaign [12] and guidelines for management of severe community acquired pneumonia [13]. These have had an impact on early recognition and treatment of septic patients, for instance by underscoring the importance of early antibiotic treatment [14]. However, important determinants of poor outcome of the disease remain to be identified to improve the management and treatment of IPD patients. We carried out a prospective observational study of risk factors for 30 and 90-day mortality among patients with IPD and sepsis in Nord-Trøndelag county, Norway from 1993 to 2011.

\section{Methods}

\section{Setting and population}

Nord-Trøndelag is a county in Central Norway with a population of approximately 134000 . It has both coastal and inland municipalities and consists of rural areas as well as small cities, but lack big cities. It is served by two community hospitals, Namsos Hospital and Levanger Hospital. The closest tertiary referral hospital is St Olav's Hospital, Trondheim University Hospital. We included all patients $\geq 16$ years of age with IPD confirmed by growth of S.pneumoniae in blood culture or cerebrospinal fluid at
Levanger Hospital between 1993 and 2011, and at Namsos Hospital between 1999 and 2011. Residents of NordTrøndelag with IPD detected at St Olav's Hospital between 1993 and 2011 were also included if they participated in a population survey (the HUNT-2 Survey) between 1995 and 1997 [15].

BACTEC 9240 (Becton Dickinson Diagnostic Instrument Systems, Sparks, MD) was used for blood culture testing until the beginning of 2010 and thereafter the Bactec FX [16]. Spinal fluid samples were cultured on chocolate plates incubated in an atmosphere of $5 \% \mathrm{CO} 2$ for 2-3 days. S. pneumoniae was identified by typical colony morphology, microscopic morphology, and optochin sensitivity. Antibiotic susceptibility testing and screening for penicillin resistance (oxacillin $1 \mu \mathrm{g}$ disk) were performed by the disc-diffusion method (Oxoid, UK). Penicillin MIC determination (E test, AB Biodisk, Sweden) was performed in all pneumococcal isolates. Antimicrobial susceptibility was categorized according to breakpoints from EUCAST (www.eucast.org). Penicillin non-susceptibility was categorized using the breakpoint for meningitis cases ( $\mathrm{MIC}>0.064 \mathrm{mg} / \mathrm{l})$.

\section{Serotyping}

S. pneumoniae isolates from cases of IPD were forwarded to the National Reference Laboratory for Pneumococci at the Norwegian Institute of Public Health. Isolates were confirmed as pneumococci, and serotyped by the Quellung reaction using serotype-specific antisera (Statens Seruminstitut, Copenhagen, Denmark) [17].

\section{Patient characteristics}

All patients with positive blood cultures were prospectively registered and clinical information was extracted retrospectively from the patients' hospital records. All data were collected using a standardized data retrieval form assessing patients' characteristics, comorbid conditions, results of investigations and treatment. The data collection was carried out by trained research nurses and all registered data was secondarily assessed by an infectious disease consultant or the first author of the study. An episode of bloodstream infection was defined as the presence of one or more microorganism(s) in blood culture along with clinical evidence of infection. If a patient had more than one episode of $S$. pneumoniae bacteremia during the study period, only the first was included.

The setting of infection was classified as hospitalacquired (HA), healthcare- associated (HCA) or community-acquired (CA) as defined by Friedman et al. [18]. Patients who were hospitalized for two or more days during the 30 days prior to the infection were classified as having a HCA infection, in keeping with the definition used by Siegman-Igra et al. [19]. The number and severity of combined comorbid conditions were 
assessed according to the Charlson weighted Comorbidity Index (CCI) [20]. Case fatality rate was measured as all-cause mortality within day 30 and 90 . By using the 11-digit unique identification number of all Norwegian citizens, electronic hospital records in Norway are updated with mortality data from the Norwegian population registry so that mortality data after discharge from hospital can be reliably assessed [21, 22].

\section{Severity of disease}

Severity of disease (sepsis without organ failure, severe sepsis and septic shock) was determined according to the 2001 International Sepsis Conference definition [23]. We defined sepsis as documented bloodstream infection and two or more of the following: temperature $\geq 38.3^{\circ} \mathrm{C}$ or $<36.0{ }^{\circ} \mathrm{C}$, heart rate $>90$ beats $/ \mathrm{min}$, respiratory rate $>20 /$ min or $\mathrm{PaCo} 2<4.3 \mathrm{kPa}$ or mechanical ventilation due to acute respiratory failure, glucose $>7.7 \mathrm{mmol} / \mathrm{l}$ in the absence of diabetes, leucocytes $>12 \times 10^{9} / 1$ or $<4 \times$ $10^{9} / \mathrm{l}$, elevated CRP or procalcitonin, acute hypotension (systolic BT $<90 \mathrm{mmHg}$, MAP $<70 \mathrm{mmHg}$ or a fall of $\geq 40 \mathrm{mmHg}$ ), or need of fluid resuscitation $(>20 \mathrm{ml} / \mathrm{kg}$ over $24 \mathrm{~h}$ ). The specific criteria for organ dysfunction used were the same as those outlined in the 2001 International Sepsis Conference definition [23]. Any changes in disease severity were registered. The most severe deterioration of organ function was noted and used to define whether the patient had sepsis, severe sepsis or septic shock. For those who did not show any evidence of deterioration during the episode, the day of positive blood culture was registered as the day with the most severe affection. Severity of disease was also assessed by the Pitt bacteremia score [24].

\section{Focus of infection}

Lower respiratory tract focus was diagnosed with clinical signs of respiratory infection and positive radiologic findings. Reported signs of infection along with focal growth of the same microbe as in the blood culture was taken as a confirmation of skin, soft tissue, joint or surgical infection. Meningitis was diagnosed with clinical signs and growth of the microbe in the cerebrospinal fluid. Upper respiratory tract infection was defined as otitis media or sinusitis. Otitis media was diagnosed as inflammation and accumulation of infected fluid of the middle ear and fever. Sinusitis was defined as inflammation of the sinuses, fever, pain in the face or headache and the presence of thick nasal mucus. An abscess was defined as a localized collection of pus in a tissue together with bacteremia. An unknown focus of infection was assigned when none of the criteria for ascertaining a focus were met.

\section{Initial antibiotic treatment}

Appropriateness of initial antibiotic therapy (AIAT) was evaluated: initial therapy had to be given (1) intravenously in correct doses and timely after the blood culture specimen was obtained ( $<6 \mathrm{~h}$ or $>6 \mathrm{~h})$, (2) with a regimen that was active in vitro against the microbe(s) isolated from blood culture(s).

\section{Statistical analysis}

Data was analyzed using SPSS for Windows (Version 21, Armonk, NY: IBM Corp) and STATA version 13 (Stata Corp LP, College Station, Texas). The associations between clinical characteristics and 30 and 90-day mortality were investigated using logistic regression analysis where odds ratios (ORs) with $95 \%$ confidence intervals (CI) were estimated. In addition, we studied the association between clinical characteristics and severe sepsis/ septic shock as an outcome, in order to identify groups of patients particularly vulnerable to develop severe disease and need a higher level of follow-up and care. All associations were estimated both unadjusted and adjusted for potential confounders, chosen based on prior knowledge of factors that may influence both the exposure variable under interest and the risk of the outcome (death or sepsis severity). All adjusted analyses were adjusted for sex and age group ( $\leq 70,70-79$ and $\geq 80$ years). The association of the setting of infection, focus, severity, and time period were additionally adjusted for comorbid conditions using three categories of the CCI $(0,1-2$ and $\geq 3)$. Adjusted 30 - and 90 -day mortality risks and risk of severe sepsis/shock were estimated from the logistic regression model by using the margins postestimation command in Stata. For ordinal variables, we tested for linear trend across categories by using the categories as a continuous variable in the logistic regression model.

\section{Results}

We identified 414 patients with IPD during the study period. All met the sepsis criteria. The mean age was 67 years and $52.9 \%$ were male. In $78.1 \%$ of the patients, the infection was community-acquired, $17.6 \%$ of infections were healthcare-associated and $4.3 \%$ were hospitalacquired. The all-cause mortality rate was $12.3 \%$ at 30 days and $16.7 \%$ at 90 days (Table 1 ). A CCI-score of 0 was registered in 144 patients $(34.8 \%)$, whereas $1-2$ in $190(45.9 \%)$ and $\geq 3$ in $80(19.3 \%)$ of the patients (Table 1).

The 30-day mortality risk increased by age ( $\mathrm{p}$ for trend $<0.001$ ). The 30 -day mortality risk was 3 fold higher for patients aged 80 years or older $(24.9,95 \%$ CI $16.4-$ $33.4 \%)$ compared to patients aged under 70, (8.0, $95 \% \mathrm{CI}$ $3.5-12.4 \%$ ) (Table 2). A positive association with age was 
Table 1 Patient and infection characteristics

\begin{tabular}{|c|c|}
\hline Characteristic & $\mathrm{N}(\%)$ \\
\hline Patients included & $414(100)$ \\
\hline \multicolumn{2}{|l|}{ All-cause mortality } \\
\hline 30-days & $51(12.3)$ \\
\hline 90-days & $69(16.7)$ \\
\hline \multicolumn{2}{|l|}{ Sex } \\
\hline Female & $195(47.1)$ \\
\hline Male & $219(52.9)$ \\
\hline \multicolumn{2}{|l|}{ Acquisition } \\
\hline Community-acquired & $323(78.1)$ \\
\hline Healthcare-associated & $73(17.6)$ \\
\hline Hospital-acquired & $18(4.3)$ \\
\hline \multicolumn{2}{|l|}{ Age category } \\
\hline$<50$ & $69(16.7)$ \\
\hline $50-69$ & $131(31.6)$ \\
\hline 70-79 & $104(25.1)$ \\
\hline$\geq 80$ & $110(26.6)$ \\
\hline \multicolumn{2}{|l|}{ Comorbid conditions } \\
\hline Malignancy & $90(21.7)$ \\
\hline Renal failure & $24(5.8)$ \\
\hline Diabetes mellitus & $51(12.3)$ \\
\hline Hypertension & $98(23.7)$ \\
\hline Coronary heart disease & $84(20.3)$ \\
\hline Heart failure & $40(9.7)$ \\
\hline Chronic pulmonary disease & $96(23.2)$ \\
\hline Cerebral ischemic disease & $40(9.7)$ \\
\hline \multicolumn{2}{|l|}{ Charlson comorbidity index (CCI) } \\
\hline 0 & $144(34.8)$ \\
\hline $1-2$ & $190(45.9)$ \\
\hline$\geq 3$ & $80(19.3)$ \\
\hline \multicolumn{2}{|l|}{ Severity of sepsis } \\
\hline Sepsis without organ failure & $267(64.5)$ \\
\hline Severe sepsis & $110(26.6)$ \\
\hline Septic shock & $37(8.9)$ \\
\hline \multicolumn{2}{|l|}{ Pitt bacteremia score } \\
\hline 0 & $126(30.4)$ \\
\hline 1 & $131(31.6)$ \\
\hline 2 & $72(17.4)$ \\
\hline$\geq 3$ & $85(20.6)$ \\
\hline Focus of infection & N (\%) \\
\hline Abdomen & $4(1.0)$ \\
\hline Lower respiratory tract & $344(83.0)$ \\
\hline Upper respiratory tract & $11(2.7)$ \\
\hline Skin/soft tissue/abscess/myositis & $13(3.1)$ \\
\hline Meningitis & $26(6.3)$ \\
\hline Unknown & $16(3.9)$ \\
\hline
\end{tabular}

Table 1 Patient and infection characteristics (Continued)

\begin{tabular}{ll}
\hline Time period & \\
1993-2002 & $134(32.4)$ \\
2003-2011 & $280(67.6)$ \\
Management & \\
Treatment in Intensive Care Unit(ICU) & $136(32.9)$ \\
Treatment with vasopressors & $40(9.6)$ \\
Ventilator treatment & $16(3.9)$ \\
Penicillin-sensitive Streptococcus pneumoniae & $397(97.8)^{\mathrm{a}}$ \\
\hline
\end{tabular}

${ }^{\mathrm{a} W e}$ had information about 406 isolates of S.pneumoniae

also seen for the 90-day mortality risk (Additional file 1: Table S1).

Females had lower 30-day mortality risk than males $8.9 \%$ vs $15.4 \%$ (OR $0.5195 \%$ CI 0.27-0.97) (Table 2). This association was nearly unchanged but less precise after adjusting for comorbid disease (OR $0.5595 \%$ CI $0.29-1.05)$ and after adjusting for severity of sepsis on admission, time to antibiotics and place of acquisition (OR $0.5495 \%$ CI 0.26-1.10). A similar gender association was also seen for 90-day mortality risk. (Additional file 1: Table S1).

The initial antibiotic treatment was mainly penicillin, which is the standard empiric treatment for communityacquired pneumonia in Norway or penicillin in combination with aminoglycoside, which is the standard empiric treatment for severe community-acquired pneumonia or sepsis of unknown origin period. Overall, $85.5 \%$ received antibiotics according to national guidelines [25, 26], $97.8 \%$ of the pneumococcal isolates were sensitive to penicillin. Of the 406 isolates, 397 were sensitive to penicillin, seven were intermediate and two were resistant. We did not have information about eight samples. Within $6 \mathrm{~h}, 68.8 \%$ of the patients received antibiotic treatment, $18.6 \%$ received treatment after $6 \mathrm{~h}$ and data on timing of treatment were lacking for $12.6 \%$ of the patients. Patients who received antibiotics before $6 \mathrm{~h}$ had a 30-day mortality risk of $11.9 \%$ (95 \% CI 8.5$15.3 \%)$, which was similar to patients who received antibiotics after $6 \mathrm{~h}$ (13.2, 95 \% CI 8.1-18.4 \%) (Table 3).

With regard to severity of disease, $64.5 \%$ of the patients had sepsis without organ failure, $26.6 \%$ had severe sepsis and $8.9 \%$ had septic shock. In patients with sepsis with no organ failure the 30- day mortality risk was $5.4 \%$ (95 \% CI 2.7-8.0 \%), the 30-day mortality risk was almost 4-fold higher for patients with severe sepsis (20.2, $95 \%$ CI 13.5-27.4 \%) and more than 6-fold higher for patients with septic shock (35.0, 95 \% CI 21.6-49.0\%) (Table 3). A similar association was seen for 90 -day mortality risk (Additional file 1: Table S2).

An increase in mortality risk according to severity was also observed for the Pitt bacteremia score, especially for 
Table 2 30-day all-cause mortality in relation to patient characteristics prior to infection

\begin{tabular}{|c|c|c|c|c|c|c|c|}
\hline \multirow[t]{2}{*}{ Characteristic } & \multirow{2}{*}{$\begin{array}{l}\text { No. of deaths } \\
\text { within } 30 \text { days }\end{array}$} & \multirow{2}{*}{$\begin{array}{l}\text { 30-day mortality } \\
\text { within category (\%) }\end{array}$} & \multicolumn{5}{|c|}{ Age- and sex-adjusted } \\
\hline & & & Odds ratio & $95 \% \mathrm{Cl}$ & $\mathrm{p}$ & Mortality risk (\%) & $95 \% \mathrm{Cl}$ \\
\hline \multicolumn{8}{|l|}{ Age (years) } \\
\hline$<70$ & 12 & 6.0 & 1 & Reference & & 8.0 & $3.5-12.4$ \\
\hline 70-79 & 15 & 14.4 & 2.72 & $1.21-6.07$ & 0.02 & 14.0 & $7.4-20.6$ \\
\hline$\geq 80$ & 24 & 21.8 & 5.57 & $2.63-11.78$ & $<0.001$ & 24.9 & $16.4-33.4$ \\
\hline$p$ for trend & & & & & $<0.001$ & & \\
\hline \multicolumn{8}{|l|}{ Sex } \\
\hline Male & 33 & 15.1 & 1 & Reference & & 15.4 & $10.8-20.1$ \\
\hline Female & 18 & 9.2 & 0.51 & $0.27-0.97$ & 0.040 & 8.9 & $5.0-12.8$ \\
\hline \multicolumn{8}{|l|}{ Charlson Comorbidity Index (CCl) } \\
\hline 0 & 12 & 8.4 & 1 & Reference & & 12.9 & $6.3-19.5$ \\
\hline $1-2$ & 20 & 10.5 & 0.67 & $0.30-1.51$ & 0.34 & 9.3 & $5.5-13.1$ \\
\hline$\geq 3$ & 19 & 23.8 & 1.54 & $0.66-3.60$ & 0.32 & 18.1 & $10.8-25.4$ \\
\hline $\mathrm{p}$ for trend & & & & & 0.11 & & \\
\hline \multicolumn{8}{|l|}{ Comorbidities $^{a}$} \\
\hline Malignant disease & 18 & 20.0 & 1.61 & $0.82-3.13$ & 0.15 & 16.1 & $9.2-23.0$ \\
\hline Renal failure & 6 & 25.0 & 2.25 & $0.82-6.21$ & 0.11 & 21.7 & $7.0-36.4$ \\
\hline Diabetes mellitus & 9 & 17.6 & 1.44 & $0.64-3.28$ & 0.43 & 15.7 & $6.1-24.9$ \\
\hline Hypertension & 22 & 22.4 & 2.35 & $1.22-4.54$ & 0.01 & 19.3 & $12.0-26.6$ \\
\hline Coronary heart disease & 16 & 19.0 & 1.05 & $0.52-2.11$ & 0.75 & 12.6 & $6.7-18.6$ \\
\hline Heart failure & 6 & 15.0 & 0.62 & $0.23-1.62$ & 0.35 & 8.7 & $1.9-15.4$ \\
\hline Chronic pulmonary disease & 10 & 10.4 & 0.73 & $0.34-1.55$ & 0.49 & 10.0 & $4.3-15.8$ \\
\hline Cerebral ischemic disease & 7 & 17.5 & 1.03 & $0.40-2.50$ & 0.93 & 12.4 & $6.7-18.6$ \\
\hline
\end{tabular}

${ }^{\mathrm{a}}$ Those not having the condition were used as reference category

patients with Pitt bacteremia score $\geq 3$ (Table 3 and Additional file 1: Table S2).

There was no convincing association between CCIscore and 30-day mortality but patients with a CCI-score $\geq 3$ had a twofold higher 90-day mortality risk (27.2, $95 \%$ CI 18.8-35.7\%) compared to patients with a CCI score of 0 (12.9, 95 \% CI 6.3-19.5 \%) (Additional file 1: Table S1). With regard to individual comorbidities, hypertension was associated with increased 30-day mortality risk (OR 2.35, $95 \%$ CI 1.22-4.54) (Table 2). A similar result was observed for 90-day mortality (Additional file 1: Table S1).

This study was carried out over a period of more than 18 years. The age, sex and comorbidity adjusted 30- day mortality risk did not differ between the first (1993-2002) and second (2003-2011) periods of the study $(13.5,95 \%$ CI 7.9-19.2 \%) vs (11.8, 95 \% CI 8.2-15.3\%) (Table 3). The 90-day mortality risk tended to be higher in the first than the second period (20.6, $95 \%$ CI 14.2-27.0\%) vs (14.9, 95 \% CI 11.0-18.7 \%) (Additional file 1: Table S2).

Patients with heart failure had the highest risk of acquiring severe sepsis or septic shock (58.1, $95 \%$ CI 33.4-73.5\%) and patients with renal failure had a risk of $53.5 \%$ (95 \% CI 33.4-73.5\%). The risk of acquiring severe sepsis or septic shock was lower in the second half of the study with an adjusted risk of $29.9 \%$ (95\% CI 24.5 - $35.2 \%$ ) versus $47.3 \%$ (95 \% CI 38.9-55.7\%) (Table 4).

Serotype-information was available in 373 patients (90\%). Before introduction of the 7 -valent pneumococcal conjugate vaccine (PCV 7) in the childhood immunization in 2006, the most frequent serotypes were 4, 14, 1, 9 and 3 . In the period 2007 to 2011 the most frequent serotypes were 7 F, 22 F, 4, 3 and 19A, of which only serotype 4 is included in PCV 7 (Additional file 2: Figure S1-3).

\section{Discussion}

In our study, there were strong positive associations of age and disease severity with case fatality rate in patients with IPD and sepsis. We did not observe a reduction in mortality risk over the time of our study.

IPD is a severe disease that predominantly occurs in elderly people with comorbid disease [27]. The mortality risk was higher in patients $>80$ years of age than in younger patients. First, the clinical presentation of sepsis in elderly patients may differ from younger patients. Unspecific symptoms, like abdominal pain, confusion or 
Table 330 day all-cause mortality in relation to disease acquisition, severity, focus and time period

\begin{tabular}{|c|c|c|c|c|c|c|c|}
\hline \multirow[t]{2}{*}{ Characteristic } & \multirow{2}{*}{$\begin{array}{l}\text { No. of deaths } \\
\text { within } 30 \text { days }\end{array}$} & \multirow{2}{*}{$\begin{array}{l}\text { 30-day mortality within } \\
\text { category }(\%)\end{array}$} & \multicolumn{5}{|c|}{ Age-, sex- and comorbidity-adjusted ${ }^{\mathrm{a}}$} \\
\hline & & & Odds ratio & $95 \% \mathrm{Cl}$ & $p$ & Mortality risk (\%) & $95 \% \mathrm{Cl}$ \\
\hline \multicolumn{8}{|l|}{ Place of acquisition } \\
\hline Community acquired & 34 & 10.6 & 1 & Reference & & 10.9 & $7.6-14.3$ \\
\hline Health-care associated & 12 & 17.0 & 1.58 & $0.65-3.20$ & 0.35 & 14.7 & $6.9-22.4$ \\
\hline Hospital acquired & 5 & 27.8 & 3.13 & $0.92-10.43$ & 0.07 & 25.2 & $6.7-43.9$ \\
\hline \multicolumn{8}{|l|}{ Severity } \\
\hline Sepsis without organ failure & 14 & 5.2 & 1 & Reference & & 5.4 & $2.7-8.02$ \\
\hline Severe sepsis & 23 & 21.0 & 5.19 & $2.44-11.10$ & $<0.001$ & 20.2 & $13.5-27.4$ \\
\hline Septic shock & 14 & 37.8 & 13.58 & $5.32-34.60$ & $<0.001$ & 35.0 & $21.6-49.0$ \\
\hline$p$ for trend & & & & & $<0.001$ & & \\
\hline \multicolumn{8}{|l|}{ Pitt bacteremia score } \\
\hline 0 & 12 & 9.5 & & Reference & & 9.4 & $4.5-14.3$ \\
\hline 1 & 7 & 5.4 & 0.47 & $0.17-1.28$ & 0.14 & 4.9 & $1.4-8.3$ \\
\hline 2 & 8 & 11.1 & 1.45 & $0.53-3.94$ & 0.47 & 12.7 & $4.9-20.5$ \\
\hline$\geq 3$ & 24 & 28.2 & 5.10 & $2.12-11.65$ & $<0.001$ & 29.8 & $20.7-39.0$ \\
\hline$p$ for trend & & & & & $<0.001$ & & \\
\hline \multicolumn{8}{|l|}{ Time period } \\
\hline 1993-2002 & 17 & 12.7 & 1 & Reference & & 13.5 & 7.9-19.2 \\
\hline 2003-2011 & 34 & 12.1 & 0.77 & $0.43-1.61$ & 0.59 & 11.8 & $8.2-15.3$ \\
\hline \multicolumn{8}{|l|}{ Time to antibiotics ${ }^{\mathrm{b}}$} \\
\hline$<6$ hours & 33 & 14.1 & 1 & Reference & & 11.9 & $8.5-15.3$ \\
\hline$>6$ hours & 18 & 11.5 & 1.17 & $0.58-2.34$ & 0.65 & 13.2 & $8.1-18.4$ \\
\hline
\end{tabular}

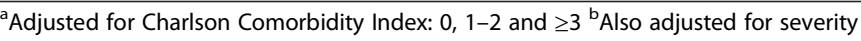

specific organ symptoms like dyspnea, can delay the sepsis diagnosis and initiation of therapy [28]. Indeed a recent Norwegian study found that antimicrobial therapy was less in compliance with current recommendations and more delayed in elderly patients with sepsis [29]. Second, ageing of the immune system and impaired effect of vaccine also contribute to the mortality risk [27]. Also, elderly patients may not be admitted to the intensive care unit due to high comorbidity burden or an end of life care situation.

We assessed whether individual prior comorbid conditions influenced the risk of acquiring severe sepsis or septic shock and found this risk was highest for patients with heart failure and renal failure. This is probably because of reduced residual capacity to meet the hemodynamic burden associated with severe sepsis $[27,30]$. Interestingly, we found that females had lower mortality risk than males for both 30-day and 90-day mortality. Males had a higher comorbidity burden than females, but adjusting for comorbid disease did not change the risk estimate, neither did adjusting for severity on admission, place of acquisition or time for antibiotic treatment. Differences in alcohol and smoking habits may have influenced the mortality risk but we could not examine that possibility $[31,32]$.

A high proportion of the patients in our study received antibiotics according to guidelines and within the first $6 \mathrm{~h}$ after admission in the hospital or in the hospital within $6 \mathrm{~h}$ after suspected invasive infection and sepsis. There was no significant reduction in mortality when comparing whether the patients received antibiotics within $6 \mathrm{~h}$ or after $6 \mathrm{~h}$. Confounding by indication is a complicating factor when assessing the effect of antibiotic treatment and outcome. It is possible that those with more severe illness received antibiotic treatment earlier. We adjusted for age, sex, comorbid disease burden and severity but there may still be unadjusted differences between the groups. In addition, it is likely that there were variations in time from how long the patients had suffered from the infection prior to admission since almost $80 \%$ of the patients had community- acquired infection.

As expected, we saw a change in the serotype pattern before and after 2006. The three most frequent serotypes after 2006 were 7 F, 22 F, and 3. A metaanalysis from 2010 found that serotypes 3,6A, 6B, $9 \mathrm{~N}$ and $19 \mathrm{~F}$ were 
Table 4 Proportion of severe sepsis/septic shock according to prior patient characteristics and infection-related characteristics

\begin{tabular}{|c|c|c|c|c|c|c|c|}
\hline \multirow[t]{2}{*}{ Characteristic } & \multirow{2}{*}{$\begin{array}{l}\text { No with severe sepsis } \\
\text { or septic shock }\end{array}$} & \multirow{2}{*}{$\begin{array}{l}\text { Severe sepsis or septic } \\
\text { shock in category (\%) }\end{array}$} & \multicolumn{5}{|c|}{ Age-, sex- and comorbidity ${ }^{\mathrm{a}}$-adjusted } \\
\hline & & & Odds ratio & $95 \% \mathrm{Cl}$ & $p$ & $\begin{array}{l}\text { Risk of severe sepsis } \\
\text { or septic shock (\%) }\end{array}$ & $95 \% \mathrm{Cl}$ \\
\hline \multicolumn{8}{|l|}{ Age (years) } \\
\hline$<70$ & 73 & 36.5 & 1 & Reference & & 35.0 & $28.7-42.0$ \\
\hline $70-79$ & 33 & 31.7 & 0.81 & $0.48-1.37$ & 0.44 & 30.8 & $22.0-39.8$ \\
\hline$\geq 80$ & 41 & 37.2 & 1.24 & $0.75-2.10$ & 0.39 & 40.7 & $30.8-50.5$ \\
\hline$p$ for trend & & & & & 0.49 & & \\
\hline \multicolumn{8}{|l|}{ Sex } \\
\hline Male & 82 & 37.4 & 1 & Reference & & 37.6 & $31.2-44.0$ \\
\hline Female & 65 & 33.3 & 0.88 & $0.57-1.34$ & 0.54 & 33.1 & $26.5-39.8$ \\
\hline \multicolumn{8}{|l|}{ Charlson Comorbiditylndex (CCl) } \\
\hline 0 & 46 & 31.9 & 1 & Reference & & 33.0 & $24.7-41.2$ \\
\hline $1-2$ & 70 & 36.8 & 1.10 & $0.64-1.91$ & 0.73 & 36.5 & $29.5-43.3$ \\
\hline$\geq 3$ & 31 & 38.7 & 1.29 & $0.72-2.29$ & 0.39 & 37.7 & $26.8-48.5$ \\
\hline$p$ for trend & & & & & 0.37 & & \\
\hline \multicolumn{8}{|l|}{ Comorbidities $^{a}$} \\
\hline Malignant disease & 33 & 36.7 & 0.98 & $0.59-1.61$ & 0.94 & 35.1 & $25.2-45.1$ \\
\hline Renal failure & 16 & 66.7 & 2.20 & $0.95-5.11$ & 0.07 & 53.5 & $33.4-73.5$ \\
\hline Diabetes mellitus & 14 & 27,4 & 0.62 & $0.32-1.21$ & 0.16 & $26 . .7$ & $14.6-38.7$ \\
\hline Hypertension & 38 & 38.7 & 1.17 & $0.71-1.91$ & 0.54 & 38.2 & $28.2-48.1$ \\
\hline Coronary heart disease & 37 & 30.3 & 0.66 & $0.38-1.15$ & 0.14 & 28.3 & $18.4-38.2$ \\
\hline Heart failure & 23 & 57.5 & 2.82 & $1.39-5.74$ & 0.004 & 58.1 & $42.0-74.1$ \\
\hline Chronic pulmonary disease & 39 & 40.6 & 1.34 & $0.83-2.15$ & 0.23 & 40.7 & $30.8-50.5$ \\
\hline Cerebral ischemic disease & 12 & 30.0 & 0.69 & $0.33-1.43$ & 0.32 & 28.3 & $14.4-42.2$ \\
\hline \multicolumn{8}{|l|}{ Place of acquisition } \\
\hline Community acquired & 112 & 34.7 & 1 & Reference & & 34.6 & $29.6-40.0$ \\
\hline Health-care associated & 25 & 34.2 & 0.95 & $0.54-1.68$ & 0.87 & 33.6 & $23.0-45.7$ \\
\hline Hospital acquired & 10 & 55.6 & 2.37 & $0.88-6.39$ & 0.09 & 55.7 & $32.5-76.6$ \\
\hline \multicolumn{8}{|l|}{ Time periode } \\
\hline 1993-2002 & 63 & 47.0 & 1 & Reference & & 47.3 & $38.9-55.7$ \\
\hline $2003-2011$ & 84 & 30.0 & 0.47 & $0.30-0.72$ & 0.01 & 29.9 & $24.5-35.2$ \\
\hline
\end{tabular}

${ }^{\mathrm{a}}$ The association of age, sex, $\mathrm{CCl}$ and individual underlying comorbidities with the risk of severe sepsis or septic shock are not adjusted for comorbidity

associated with increased risk of death and these had high carrier prevalence, low invasiveness and were encapsulated in vitro [33]. We did not have the statistical power to examine differences in mortality between different serotypes. Following the introduction of PCV7 in the childhood immunization program in 2006 the incidence of IPD declined in all age groups as a result of indirect effect [17]. This decline continued after the switch to PCV13 in 2011 [7]. PCV13 has been licensed for prevention of IPD in all age-groups, and in 2013 the Norwegian Institute of Public Health updated the recommendations for use of pneumococcal vaccines to elderly and risk-groups with a recommendation to use PCV13 in addition to PPV23 for persons with a very high risk for IPD [34]. However, as the impact of PCV13 in the childhood immunization program continues to reduce circulation of PCV13 serotypes, the preventive potential of PCV13 given to adults and risk-groups is reduced over time [6].

We did not demonstrate any reduced mortality risk over the time of our study, but the risk of acquiring severe sepsis was significantly decreased in the later period. This may be for several reasons such as introduction of the pneumococcal vaccine in the childhood vaccination program which has shown to influence other age groups in the society [1] and improved patient management as part of a continuing awareness of severe infections and sepsis [35]. 
All patients had verified bacteremia and the blood culture were prospectively registered by the microbiology laboratory, which ensures a broad inclusion of all affected patients. Sepsis was diagnosed in IPD-patients independent of primary focus, microbiological etiology and host factors. This study has, however, some limitations. The clinical data were collected from hospital records at a later date which is inferior to prospective clinical assessment but detailed data were available on the majority of the patients. It is a strength of our study that all IPD cases were confirmed by growth in blood culture or, in a few cases, in cerebrospinal fluid. However, it is also possible to suffer from IPD without having verified bacteremia or growth in the cerebrospinal fluid, for example, if antibiotic treatment was given prior to blood sampling, and we could not identify and include such patients. The study was carried out only in one region in Norway and may not be generalizable, particularly outside Scandinavia. Regrettably, we did not have information on vaccine status, which would have been an interesting aspect to assess in this population.

\section{Conclusions}

In conclusion, we found that older age and higher severity of disease were important risk factors for death among patients with IPD and sepsis. We found no substantial decrease in case fatality rate during the study period. The study contributes to the understanding of IPD and sepsis in Norway.

\section{Abbreviations}

AIAT, appropriateness of initial antibiotic therapy; CA, community-acquired CCl Charlson Comorbidity Index; HA, hospital-acquired; HCA, Healthcareassociated; IPD, invasive pneumococcal disease; MIC, minimum inhibitory concentration; PCV, pneumococcal conjugate vaccine

\section{Additional files}

Additional file 1: Table S1. 90-day mortality in relation to patient characteristics prior to infection. Table S1a. 90-day mortality in relation to gender. Table S2. 90-day mortality in relation to disease acquisition, severity, focus and time period. (DOCX $22 \mathrm{~kb}$ )

Additional file 2: Figure S1. Serotypes 1993-2011. Figure S2.

Serotypes 1993-2006. Figure S3. Serotypes 2007-2011. (DOC 106 kb)

\section{Acknowledgments}

We thank the research nurses at Nord-Trøndelag Hospital Trust for their contribution to the data collection. We would also like to thank the staff at the Microbiology laboratory, Levanger hospital, for consecutively including cases.

\section{Funding}

This work was supported by the Liaison Committee between the Central Norway Regional Health Authority (RHA) and the Norwegian University of Science and Technology (NTNU): Nord-Trøndelag Hospital Trust's Fund for Research and Improvement; the Unit for Applied Clinical Research, Norwegian University of Science and Technology and the Norwegian Surveillance Program for Antimicrobial Resistance.

\section{Availability of data and materials}

From the Middle Norway Sepsis Registry we are still including patients and register data. There is extensive research going on and we are continuously publishing results. However, if necessary, we can provide the actual data used in this study.

\section{Authors' contributions}

$\AA$ A participated in design, data collection, statistical analysis, interpretation of the data and drafting of the manuscript. AM conceived the study and participated in design, data collection and drafting of the manuscript.

JP participated in design, statistical analysis, data collection and drafting of the manuscript. ADW participated in data interpretation and drafting of the manuscript. DV participated in data collection and drafting of the manuscript. BO participated in study design, statistical analysis, data interpretation and drafting of the manuscript. JKD participated in study design, data interpretation, and drafting of the manuscript. ES participated in study design, data interpretation and drafting of the manuscript. All the authors read and approved the final manuscript.

\section{Competing interests}

The authors declare that they have no competing interests.

\section{Consent for publication}

Not applicable.

\section{Ethics and consent to participate}

The study was approved by the regional committee for health and research ethics (REC-Central). Since no patient contact or intervention was carried out, the need for informed consent was waived.

\section{Author details}

${ }^{1}$ Clinic of Anaesthesia and Intensive Care, St Olav University Hospital, Trondheim, Norway. ${ }^{2}$ Department of Circulation and Medical Imaging, Norwegian University of Science and Technology, Trondheim, Norway. ${ }^{3}$ Centre of Molecular Inflammation Research Department of Cancer Research and Molecular Medicine, Norwegian University of Science and Technology, Trondheim, Norway. ${ }^{4}$ Department of Medicine, Levanger Hospital, Nord-Trøndelag Health Trust, Levanger, Norway. ${ }^{5}$ Department of Public Health, Norwegian University of Science and Technology, Trondheim, Norway. ${ }^{6}$ Department of Infectious Diseases, St Olav University Hospital Trondheim, Norway. ${ }^{7}$ Department of Endocrinology, St Olav University Hospital, Trondheim, Norway. ${ }^{8}$ Department of Chronic Disease Epidemiology, Yale School of Public Health, New Haven, CT, USA. ${ }^{9}$ Norwegian Institute of Public Health, Oslo, Norway. ${ }^{10}$ Middle Norway Sepsis Research Center, Norwegian University of Science and Technology, Trondheim, Norway. ${ }^{11}$ Faculty of medicine, Department of Circulation and Medical Imaging, Po box 8905 N-7491 Trondheim, Norway.

Received: 13 September 2015 Accepted: 7 May 2016

Published online: 23 May 2016

\section{References}

1. Muhammad RD, Oza-Frank R, Zell E, Link-Gelles R, Narayan KM, Schaffner W, et al. Epidemiology of invasive pneumococcal disease among high-risk adults since the introduction of pneumococcal conjugate vaccine for children. Clin Infect Dis. 2013:56(5):e59-67.

2. Drijkoningen $\mathrm{J}$, Rohde GG. Pneumococcal infection in adults: burden of disease. Clin Microbiol Infect. 2014;20 Suppl 5:45-51.

3. Li Y, Weinberger DM, Thompson CM, Trzcinski K, Lipsitch M. Surface charge of Streptococcus pneumoniae predicts serotype distribution. Infect and immun. 2013;81(12):4519-24

4. Dockrell DH, Whyte MK, Mitchell TJ. Pneumococcal pneumonia: mechanisms of infection and resolution. Chest. 2012;142(2):482-91.

5. Chavanet P. Pneumococcus infections: is the burden still as heavy? Med et mal infect. 2012;42(4):149-53.

6. Steens A, Vestrheim DF, de Blasio BF. Pneumococcal vaccination in older adults in the era of childhood vaccination: Public health insights from a Norwegian statistical prediction study. Epidemics. 2015;11:24-31.

7. Steens A, Bergsaker MA, Aaberge IS, Ronning K, Vestrheim DF. Prompt effect of replacing the 7-valent pneumococcal conjugate vaccine with the 
13-valent vaccine on the epidemiology of invasive pneumococcal disease in Norway. Vaccine. 2013;31(52):6232-8.

8. Koppe U, Suttorp N, Opitz B. Recognition of Streptococcus pneumoniae by the innate immune system. Cell Microbiol. 2012;14(4):460-6.

9. van der Poll T, Opal SM. Pathogenesis, treatment, and prevention of pneumococcal pneumonia. Lancet. 2009:374(9700):1543-56.

10. van Hoek AJ, Andrews N, Waight PA, Stowe J, Gates P, George R, et al. The effect of underlying clinical conditions on the risk of developing invasive pneumococcal disease in England. J Infect. 2012;65(1):17-24.

11. Ludwig E, Bonanni P, Rohde G, Sayiner A, Torres A. The remaining challenges of pneumococcal disease in adults. Eur Respir Rev. 2012;21(123):57-65.

12. Dellinger RP, Levy MM, Rhodes A, Annane D, Gerlach H, Opal SM, et al. Surviving sepsis campaign: international guidelines for management of severe sepsis and septic shock: 2012. Crit Care Med. 2013;41(2):580-637.

13. Brown SM, Jones BE, Jephson AR, Dean NC. Validation of the Infectious Disease Society of America/American Thoracic Society 2007 guidelines for severe community-acquired pneumonia. Crit Care Med. 2009;37(12):3010-6.

14. Bakker J, Perner A, Timsit JF. Evaluation of 7.5 years of surviving sepsis campaign guidelines. Intensive Care Med. 2014;41(1):151-3.

15. Krokstad S, Langhammer A, Hveem K, Holmen TL, Midthjell K, Stene TR, et al. Cohort profile: the HUNT study, Norway. Int J Epidemiol. 2013;42(4):968-77.

16. Nolte FS, Williams JM, Jerris RC, Morello JA, Leitch CD, Matushek S, et al. Multicenter clinical evaluation of a continuous monitoring blood culture system using fluorescent-sensor technology (BACTEC 9240). J Clin Microbiol. 1993;31(3):552-7.

17. Vestrheim DF, Hoiby EA, Bergsaker MR, Ronning K, Aaberge IS, Caugant DA. Indirect effect of conjugate pneumococcal vaccination in a $2+1$ dose schedule. Vaccine. 2010;28(10):2214-21.

18. Friedman ND, Kaye KS, Stout JE, McGarry SA, Trivette SL, Briggs JP, et al. Health care-associated bloodstream infections in adults: a reason to change the accepted definition of community-acquired infections. Ann Intern Med. 2002;137(10):791-7.

19. Siegman-Igra Y, Fourer B, Orni-Wasserlauf R, Golan Y, Noy A, Schwartz D, et al. Reappraisal of community-acquired bacteremia: a proposal of a new classification for the spectrum of acquisition of bacteremia. Clin Infect Dis. 2002:34(11):1431-9.

20. Charlson ME, Pompei P, Ales KL, MacKenzie CR. A new method of classifying prognostic comorbidity in longitudinal studies: development and validation. J Chronic Dis. 1987;40(5):373-83.

21. Mehl A, Harthug S, Lydersen S, Paulsen J, Asvold BO, Solligard E, et al. Prior statin use and 90-day mortality in Gram-negative and Gram-positive bloodstream infection: a prospective observational study. Eur J Clin Microbiol Infect Dis. 2015;34(3):609-17.

22. Paulsen J, Mehl A, Askim A, Solligard E, Asvold BO, Damas JK. Epidemiology and outcome of Staphylococcus aureus bloodstream infection and sepsis in a Norwegian county 1996-2011: an observational study. BMC Infect Dis. 2015;15:116

23. Levy MM, Fink MP, Marshall JC, Abraham E, Angus D, Cook D, et al. 2001 SCCM/ESICM/ACCP/ATS/SIS International Sepsis Definitions Conference. Intensive Care Med. 2003;29(4):530-8.

24. Rhee JY, Kwon KT, Ki HK, Shin SY, Jung DS, Chung DR, et al. Scoring systems for prediction of mortality in patients with intensive care unit-acquired sepsis: a comparison of the Pitt bacteremia score and the Acute Physiology and Chronic Health Evaluation II scoring systems. Shock. 2009:31(2):146-50.

25. Helsedirektoratet, Norway http://sites.helsedirektoratet.no/sites/ antibiotikabruk-i-sykehus/Sider/default.aspx Accessed 20 Jan 2016

26. Lindbaek $M$, et al. New guidelines for use of antibiotics in the primary health care service. Tidsskr Nor Legeforen. http://tidsskriftet.no/article/ 3016792. Accessed 2 Feb 2016

27. Naucler P, Darenberg J, Morfeldt E, Ortqvist A, Henriques NB. Contribution of host, bacterial factors and antibiotic treatment to mortality in adult patients with bacteraemic pneumococcal pneumonia. Thorax. 2013;68(6):571-9.

28. Wester AL, Dunlop O, Melby KK, Dahle UR, Wyller TB. Age-related differences in symptoms, diagnosis and prognosis of bacteremia. BMC Infect Dis. 2013;13(1):346.

29. Nygard ST, Langeland N, Flaatten HK, Fanebust R, Haugen O, Skrede S. Aetiology, antimicrobial therapy and outcome of patients with community acquired severe sepsis: a prospective study in a Norwegian university hospital. BMC Infect Dis. 2014;14:121.

30. Vincent JL, De Backer D. Circulatory shock. N Engl J Med. 2014;370(6):583.

31. Grau I, Ardanuy C, Calatayud L, Schulze MH, Linares J, Pallares R. Smoking and alcohol abuse are the most preventable risk factors for invasive pneumonia and other pneumococcal infections. Int J Infect Dis. 2014;25:59-64.

32. Huttunen R, Heikkinen T, Syrjanen J. Smoking and the outcome of infection. J Intern Med. 2011;269(3):258-69.

33. Weinberger DM, Harboe ZB, Sanders EA, Ndiritu M, Klugman KP, Ruckinger $\mathrm{S}$, et al. Association of serotype with risk of death due to pneumococcal pneumonia: a meta-analysis. Clin Infect Dis. 2010;51(6):692-9.

34. Steens A, Vestrheim DF, Aaberge IS, Wiklund BS, Storsaeter J, Riise Bergsaker $M A$, et al. A review of the evidence to inform pneumococcal vaccine recommendations for risk groups aged 2 years and older. Epidemiol Infect. 2014;142(12):2471-82

35. Gu WJ, Wang F, Bakker J, Tang L, Liu JC. The effect of goal-directed therapy on mortality in patients with sepsis - earlier is better: a meta-analysis of randomized controlled trials. Crit Care. 2014;18(5):570.

\section{Submit your next manuscript to BioMed Central and we will help you at every step:}

- We accept pre-submission inquiries

- Our selector tool helps you to find the most relevant journal

- We provide round the clock customer support

- Convenient online submission

- Thorough peer review

- Inclusion in PubMed and all major indexing services

- Maximum visibility for your research

Submit your manuscript at www.biomedcentral.com/submit
) Biomed Central 